\title{
Comportement en tension et compression des pieux de fondation ou d'ancrage des ouvrages en mer
}

\author{
Compression and behaviour \\ of foundation or anchorage piles of sea works
}

\author{
P. Le Tirant
}

Institut français du pétrole

Direction de recherche "Exploitation en mer"

L'Association de Recherche en Géotechnique Marine (ARGEMA) a effectué depuis 1978 un vaste programme d'étude du comportement de pieux expérimentaux, battus ou forés-cimentés, de dimensions moyennes (diamètre $\cong 30 \mathrm{~cm}$, fiche de 13 à 23 mètres), très instrumentés (efforts, déplacements, contraintes,...), implantés sur divers sites représentatifs des sols rencontrés couramment en mer :

Chaque pieu a été soumis à un programme important de chargements simulant l'action soit d'une houle d'amplitude constante, soit de tempête centennales.

Les résultats obtenus permettent de dégager certaines conclusions importantes sur le frottement sol-pieu en tension et compression, l'influence des chargements cycliques, l'effet de l'histoire des chargements.

Since 1978, the Marine Geotechnical Research Association (ARGEMA) has been carrying out an extensive study programme of the behaviour of experimental pile-driven or concentrated drilled piles, of average sizes (diameter $30 \mathrm{~cm}, 13$ to 23 metres jointing spoons), highly instrumented (loads, displacements, stresses,...) installed on various sites representative of soils currently encountered at sea.

Each pile has been subjected to a high load programme simulating the action of either a constant wave height swell or a centennial hurricane swell.

The results obtained enable some significant conclusions to be drawn concerning the tension and compression ground-pile friction, the influence in cyclic loads and the effect of cumulative loads.

\section{Introduction}

Le dimensionnement des pieux de fondation ou d'ancrage des ouvrages fixes (telles que les plates-formes treillis dites jackets - Fig. 1 ) et flottants (comme les plates-formes à lignes tendues, dites TLP - Fig. 2) utilisés pour l'exploitation des hydrocarbures en mer, nécessite une parfaite connaissance de leur comportement sous divers types de sollicitations monotones (statiques), cycliques et dynamiques, en tension et compression (Le Tirant, 1979).

Les pieux de fondation en mer sont toujours des pieux tubulaires, le plus souvent battus, quelquefois forés et cimentés. Leur longueur est généralement comprise entre 50 et 150 mètres et leur diamètre varie d'un mètre à plus de deux mètres. Du fait de leurs grandes longueurs, la capacité portante de ces pieux est gouvernée essentiellement par le frottement latéral sol-pieu, le terme de pointe (en compression) dépassant rarement 5 à $10 \%$ de la capacité portante. Ainsi l'ingéniérie des pieux de fondation ou d'ancrage des ouvrages en mer se ramène-t-elle essentiellement au problème de l'évaluation du terme de frottement latéral, très variable suivant la nature et les

LA HOUILLE BLANCHE/N4/5-1986 


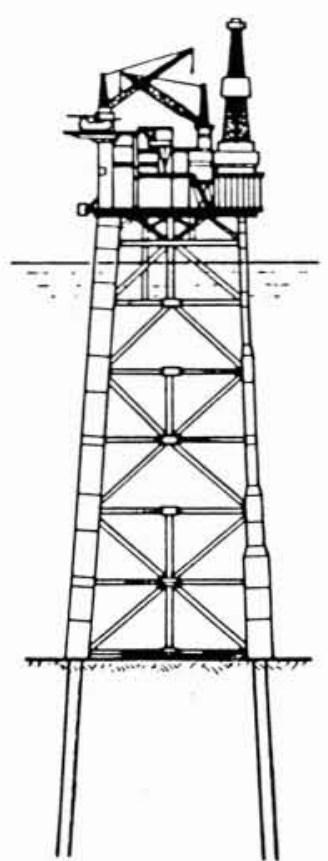

Figure 1. - Plate-forme treillis (jacket).

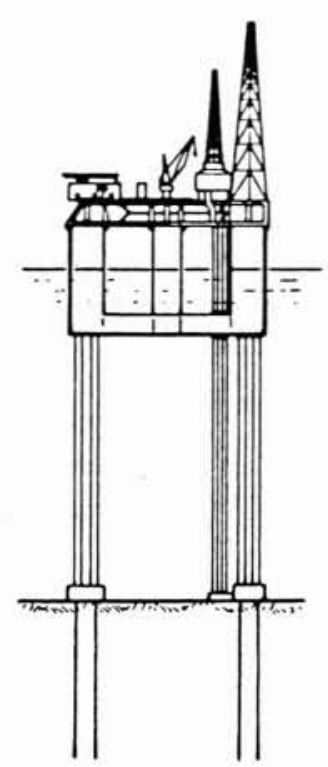

Figure 2. - Plate-forme à lignes tendues (PLT).

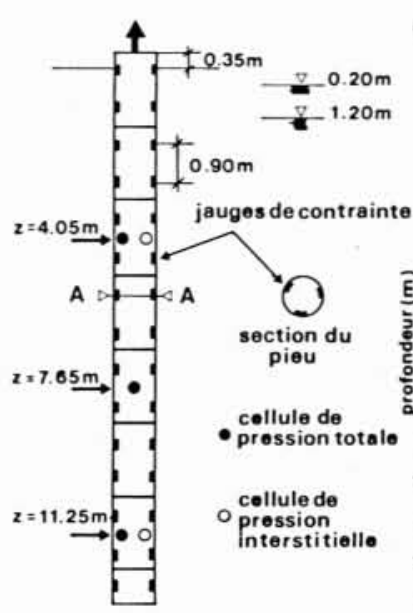

resistance de pointe au pénétromètre

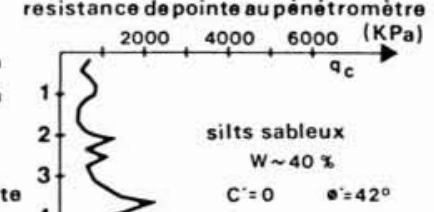

Figure 3. - Pieu battu dans un sol silteux (Plancoët)

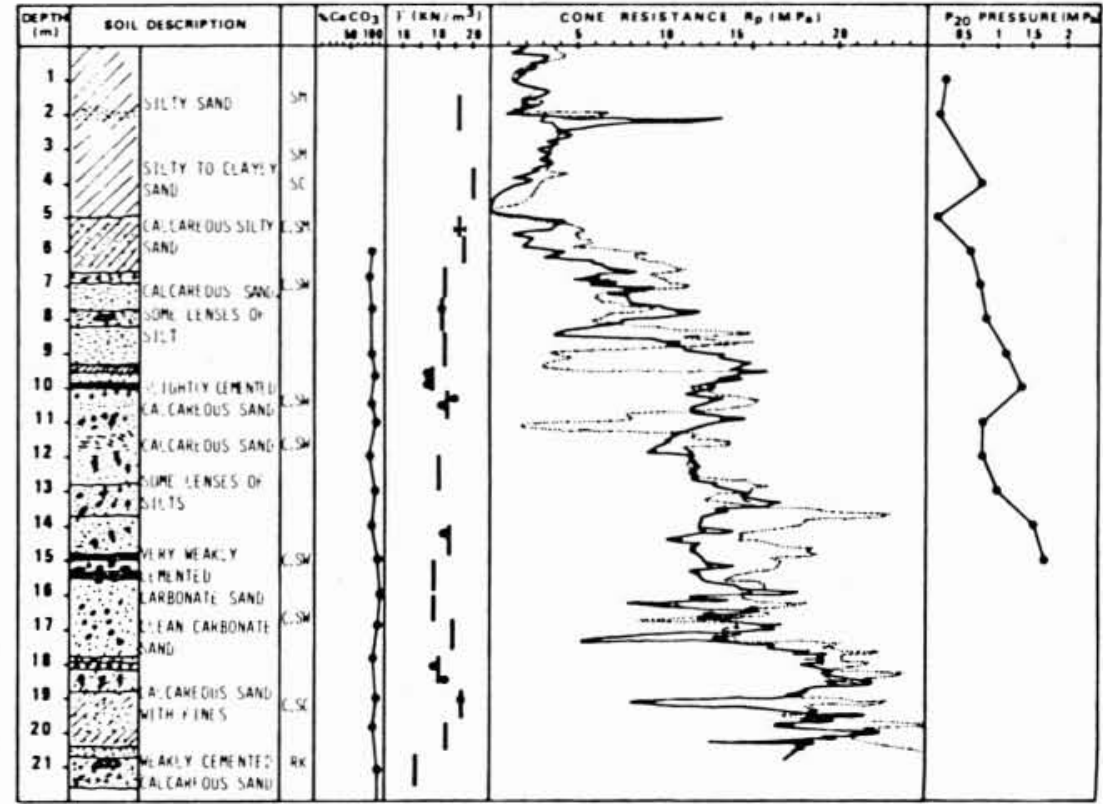

Figure 5. - Sable carbonaté (Plouasne).

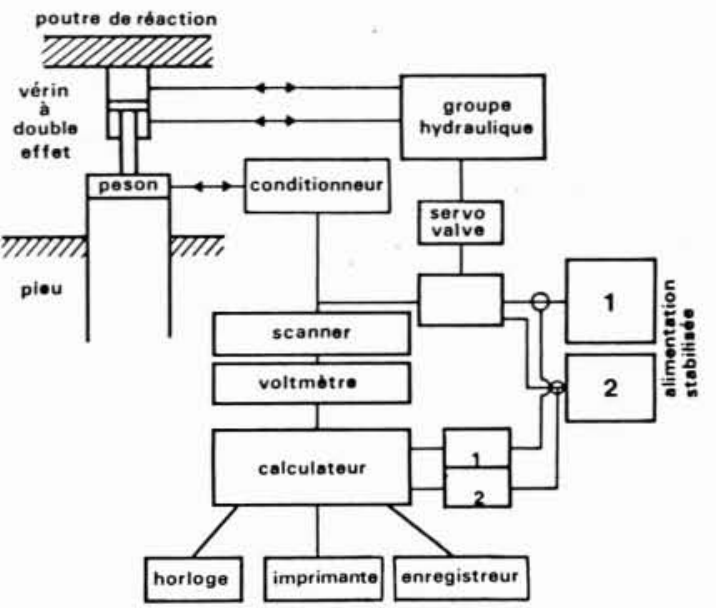

Figure 6. - Dispositif d'application des charges et d'acquisition des données. 
caractéristiques des sols, les conditions de mise en place des pieux, les types de sollicitations appliquées, etc. La dispersion des valeurs de la capacité portante suivant les différentes méthodes d'évaluation utilisées atteste l'ignorance réelle de la connaissance des phénomènes, dissimulée seulement par le choix de "coefficients de sécurité " élevés.

La nécessité d'améliorer la sécurité des ouvrages en mer par des profondeurs d'eau de plus en plus grandes, dépassant aujourd'hui 400 mètres, et d'optimiser le dimensionnement pour des raisons évidentes de coût, a conduit ces dernières années à des recherches importantes sur le comportement des pieux dans divers pays. En France, l'Association de recherche en géotechnique marine (ARGEMA) $\left({ }^{*}\right)$ a effectué depuis 1978 un vaste programme d'étude du comportement de pieux chargés axialement. D'autres expérimentations sur le comportement des pieux et groupes de pieux chargés latéralement ont été opérées en collaboration avec les laboratoires des Ponts et Chaussées.

Dans cet exposé nous nous limiterons à la description sommaire et aux principaux résultats de l'expérimentation de pieux chargés axialement.

\section{1. - Description de l'expérimentation}

Des considérations évidentes d'opération et de coût conduisent à effectuer les expérimentations au moyen de pieux de dimensions réduites par rapport aux pieux réels :

- diamètre $\mathrm{D}$ de l'ordre de $30 \mathrm{~cm}$, au lieu de 1 à 2 mètres; - longueur L du pieu dans le sol de 13 à 23 mètres, à comparer aux fiches de 50 à 150 mètres;

mais, a priori, l'effet d'échelle n'affecte pas significativement les résultats dans cette gamme de dimensions.

\subsection{Pieux expérimentaux}

Quatre pieux expérimentaux ont été mis en place par battage ou par forage-cimentation dans différents types de terrains représentatifs des sols rencontrés couramment en mer :

- Pieu 1 , battu dans un silt meuble, alternativement argileux et sableux, à Plancoët (Côtes-du-Nord) (Fig. 3): - Pieu 2 , battu dans une argile silteuse très plastique, normalement consolidée, à Cran (Morbihan), (Fig. 4);

- Pieu 3 , battu dans un sable carbonaté, coquillier (falun) du tertiaire, à Plouasne au sud de Dinan (Fig. 5); - Pieu 4 , foré et cimenté, sur le même site de sable carbonaté à Plouasne.

\subsection{Instrumentation des pieux}

L'instrumentation très complète des pieux comprenait (Puech et Jézéquel, 1980) (Fig.3);

- des capteurs de force et de déplacement en tête,

- des jauges de contrainte disposés tout le long du fût, permettant d'obtenir le frottement latéral à chaque niveau et la répartition des charges le long du pieu,

- des capteurs de pression interstitielle et de contrainte totale à différents niveaux du pieu.

Dans le cas de formations argileuses, le sol environnant était préalablement instrumenté au moyen de piézomètres disposés à différents niveaux et à différentes distances du pieu.

Cette instrumentation permettait de suivre le comportement du pieu et du sol :

- durant la mise en place du pieu par battage, puis la reconsolidation du sol,

- pendant les chargements statiques et cycliques.

\subsection{Système de chargement et d'acquisition des données.}

Un dispositif automatique d'application des charges et d'acquisition des données a été développé pour obtenir les performances désirées $\left({ }^{* *}\right)$ : contrôle parfait des charges appliquées, grand nombre de voies de mesure, grande vitesse d'acquisition des données, précision des mesures. Le schéma du dispositif commandé par un petit ordinateur est représenté sur la figure 6.

La poutre de réaction, permettant de reprendre 1000 $\mathrm{KN}$ environ en tension, peut être chargée jusqu'à 800 à $1000 \mathrm{KN}$ en compression (Fig. 7). Un chassis isole la tête du pieu et l'instrumentation de surface des variations de température ambiante.

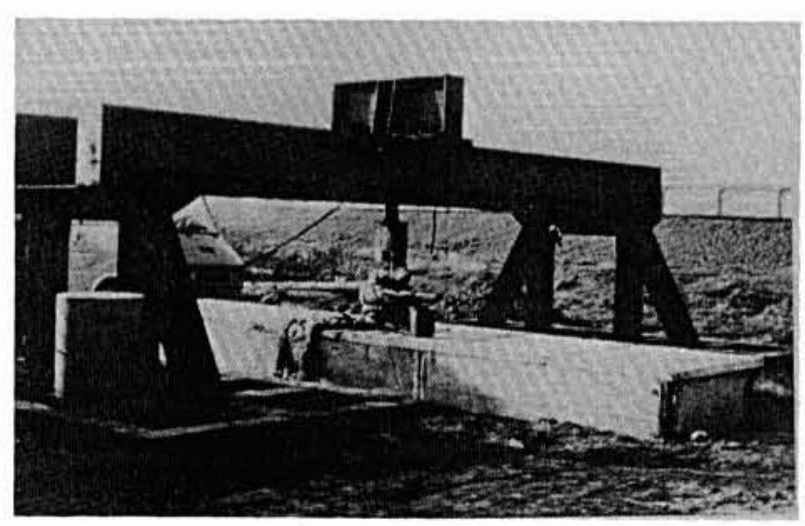

Figure 7. - Poutre de réaction

(*) L'ARGEMA regroupe IFP et IFREMER, les compagnies pétrolières CFP et $\mathrm{SNEA}(\mathrm{P})$, et plusieurs entrepreneurs du secteur parapétrolier (Boygues Offshore, CFEM, CG Doris. ETPM. Solmarine, SPIE Capag)

$\left({ }^{* *}\right)$ Les expérimentations ont été conduites avec l'assistance du Laboratoire des Ponts et Chaussées de St Brieuc. 


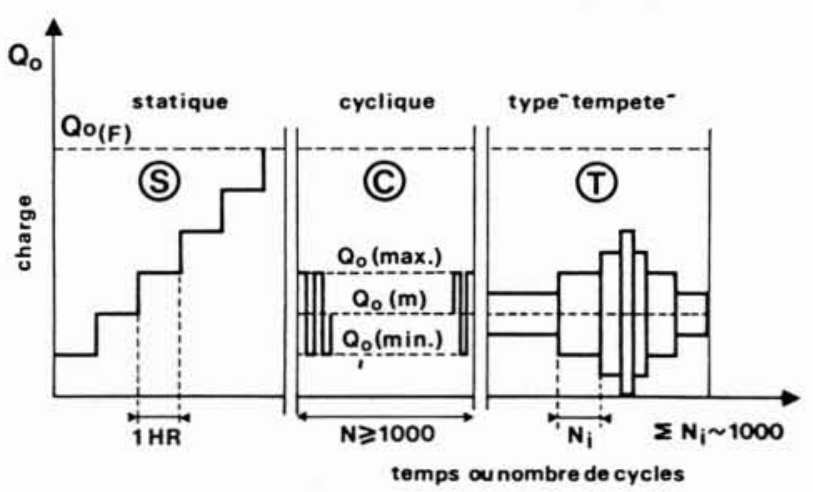

Figure 8. - Programmes de chargements appliqués.

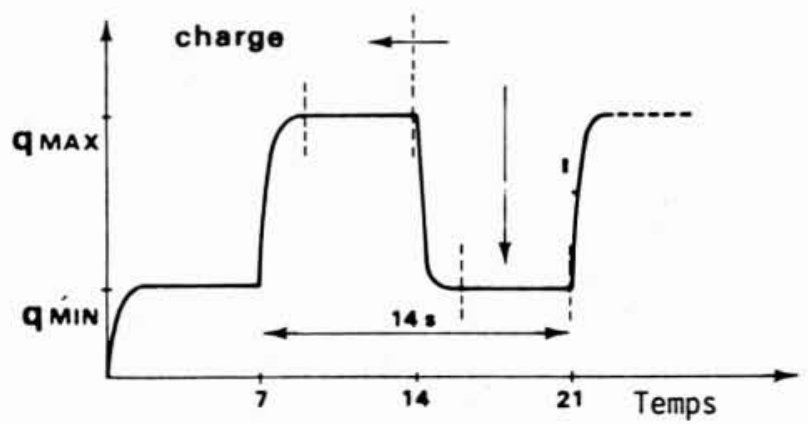

Figure 9. - Chargement cyclique.

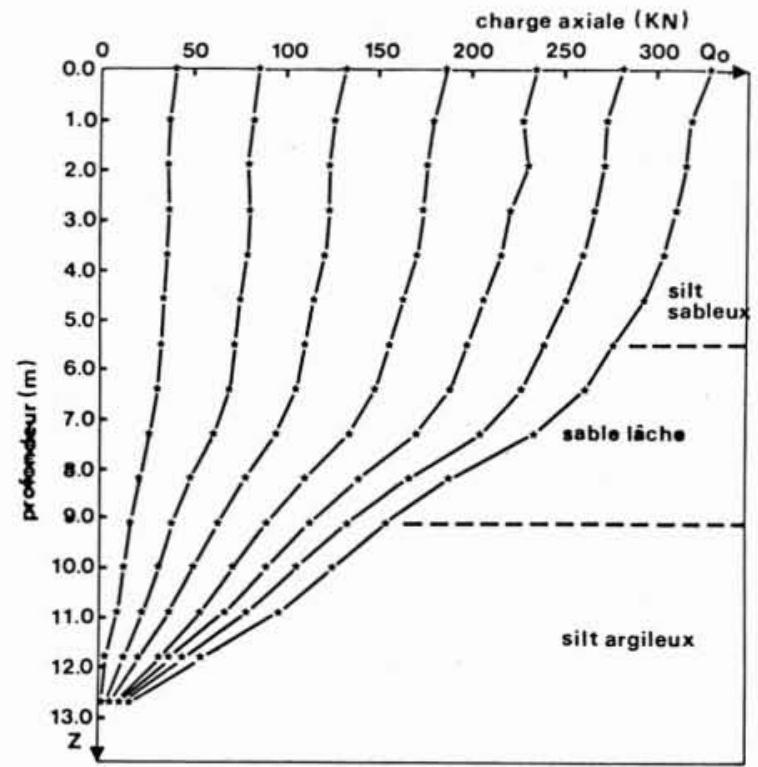

Figure 10. - Distribution de la charge le long du pieu (Plancoët).
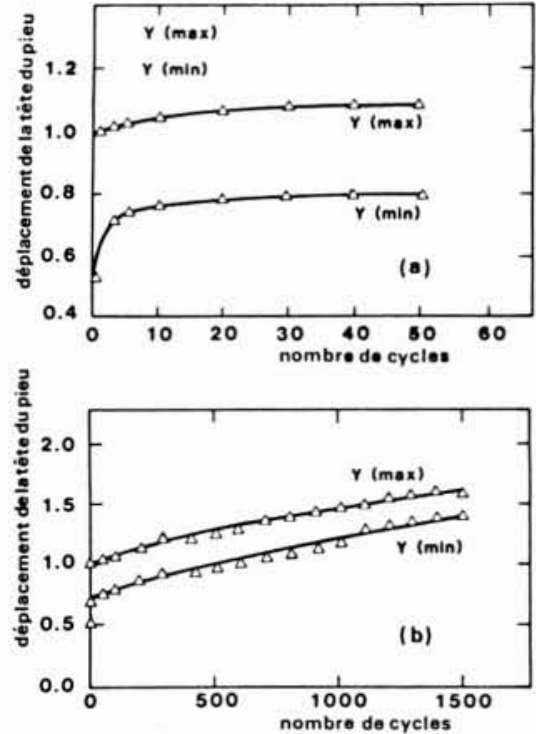

Figure 11. - Déplacements de la tête du pieu sous chargements cycliques

a) stabilisation apparente après quelques dizaines de cycles

b) comportement réel à plus long terme

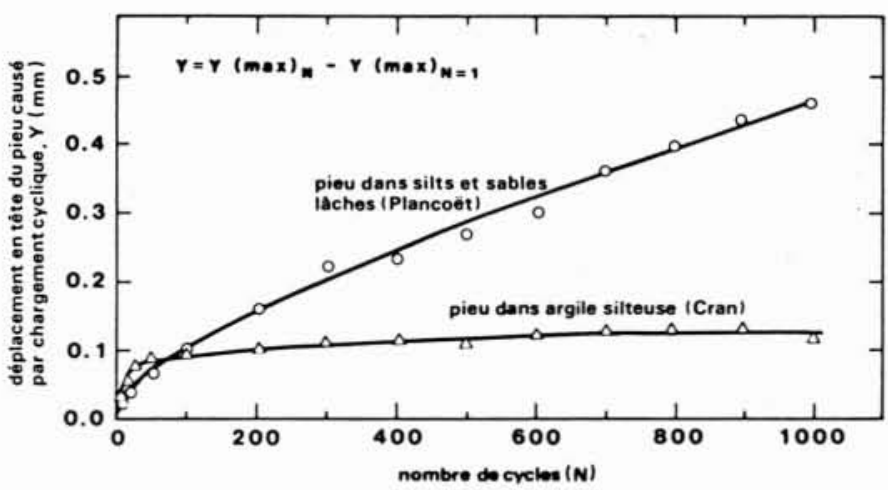

Figure 12. - Comportement des pieux sous chargement cyclique en tension.

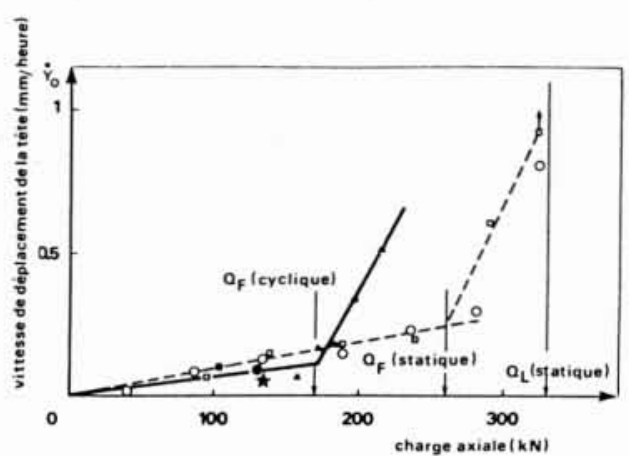

Figure 13. - Charge de fluage statique et cyclique (Plancoët).

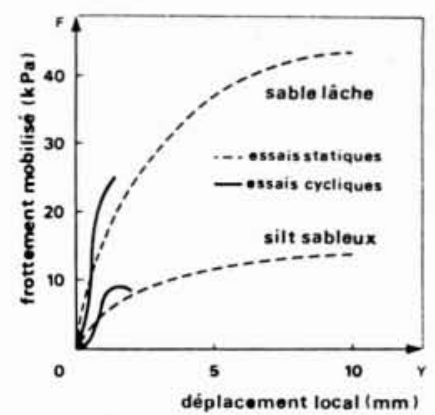

Figure 14. - Frottement latéral mobilisé sous charge statique et cyclique. 


\subsection{Programmes de chargements des pieux}

L'objectif essentiel de l'expérimentation étant la détermination du frottement latéral sol-pieu et son évolution sous chargements cycliques, la priorité a été donnée aux essais en tension (simulant le comportement des pieux d'ancrage des plates-formes à lignes tendues notamment).

Les programmes d'essais de chargements comprenaient (Fig. 8):

- des essais statiques par paliers d'une heure, à titre de référence:

- des essais cycliques à différentes amplitudes constantes, de 1000 à plusieurs milliers de cycles d'une période de 14 secondes (Fig. 9);

- des essais cycliques d'un millier de cycles d'amplitude croissante, simulant l'action de tempêtes sur le comportement des fondătions en mer.
Chaque chargement cyclique est caractérisé par :

- la charge moyenne $\mathrm{q}_{\text {moy }}$;

- l'amplitude $\Delta q$ de la charge cyclique;

ou encore :

- la charge minimale $q_{\min }=q_{\text {moy }}-\Delta q$;

- la charge maximale $q_{\max }=q_{\text {mox }}+\Delta q$.

Les déplacements correspondants en tête sont notés $y_{\mathrm{omax}}$ et $y_{\mathrm{omin}}$.

L'ordre d'application des chargements successifs a été choisi dans chaque cas en fonction des comportements observés.

Le très grand nombre d'essais de chargements effectués sur les quatre pieux expérimentaux a permis d'acquérir une somme considérable de connées sur le comportement des pieux chargés axialement. On se limite ici aux conclusions importantes suivant le type de sols, le mode de mise en place des pieux, les chargements appliqués (statiques ou cycliques, tension ou compression).

\section{2. - Comportement des pieux battus dans les sols silteux et argileux.}

Les formations silteuses et argileuses normalement consolidées se rencontrent très fréquemment en mer dans les zones deltaïques et sur la pente continentale. Les résultats acquis sur le comportement des pieux battus dans les silts lâches et les argiles peu consolidées constituent donc des repères significatifs pour l'ingénierie des pieux de fondations d'ouvrages en mer sur des sites bien identifiés.

\subsection{Pieux battus dans les silts et sables lâches.}

Le chargement statique en tension d'un pieu battu dans des couches de silts et sables lâches (Fig. 10) se traduit par un accroissement appréciable du coefficient de pression horizontale des terres qui gouverne, jusqu'à une profondeur limite, le frottement latéral des pieux dans les matériaux frottants (forations sableuses et silteuses) :

$$
f=K p_{0}^{\prime} \operatorname{tg} \delta
$$

$o / p_{0}^{\prime}$ désigne la contrainte verticale effective due au poids des terres

$\delta$ l'angle de frottement sol-pieu ( $\varphi$ étant l'angle de frottement interne)

L'évolution de K sous l'effet des chargements cycliques traduit l'influence de l'histoire des chargements préalables du pieu. Toutefois l'état final de rupture statique ne semble pas affecté par l'histoire des chargements antérieurs.

Le comportement du pieu sous chargement cyclique en tension (Puech 1982; Puech et al., 1982), avec amplitude constante, comprend deux phases (Fig. 11):

- une phase transitoire caractérisée par un accroissement rapide des déplacements en tête, dont la durée dépend de l'histoire antérieure des chargements;

- une phase de fluage (Fig. 11) dont la vitesse dépend du niveau de chargement: sous chargements de faibles niveaux, cette vitesse décroit, indiquant une tendance vers la stabilisation; au contraire sous des chargements de niveaux élevés, la vitesse croissante de fluage peut conduire à la rupture après un nombre plus ou moins grand de sollicitations cycliques, fonction à la fois de la charge moyenne $q_{\text {moy }}$ et de l'amplitude de la charge cyclique $\Delta q$.

Sous des chargements cycliques n'affectant pas la stabilité du pieu, on observe un transfert de la charge depuis la partie supérieure vers la partie inférieure du pieu (Hoeg, 1982; Nauroy et al., 1985).

La charge critique de fluage cyclique $Q_{\text {F cycl. }}$ se trouve significativement réduite par rapport à la charge critique de fluage statique (Fig. 13):

$$
Q_{\text {F cycl }} \simeq 0.6 Q_{\text {F stal. }}
$$

Le frottement latéral maximal mobilisable sous chargement cyclique dans les silts et sables lâches apparait également inférieur d'environ 30 à $40 \%$ à celui observé sous chargement statique (Fig. 14). De plus le frottement latéral maximal peut être mobilisé pour des déplacements du pieu beaucoup plus faibles que sous chargement statique.

Les phénomènes essentiels du comportement des pieux sous chargements statiques et cycliques ont été dégagés à partir des comparaisons entre les simulations numériques, effectuées notamment à l'Institut de Mécanique de Grenoble (Boulon et Foray 1985), et les résultats des essais de pieux.

\subsection{Pieux battus dans les argiles normalement consoli- dées.}

Le frottement latéral des pieux battus dans les formations argileuses est habituellement exprimé comme une fraction de la cohésion non drainée $C_{u}$ du sol $f=\alpha C_{u}$, 
toute la difficulté résidant à la fois dans le choix de la cohésion réellement mobilisée et du coefficient $\alpha$.

En chargement statique en tension, la charge limite $Q_{t}$ comme la charge critique de fluage $Q_{r}$ d'un pieu battu dans une argile silteuse, très plastique, normalement consolidée, diminuent au fur et à mesure de l'application des sollicitations cycliques. Des périodes de repos ont pour effet de stabiliser, sinon d'augmenter, la charge limite. Ces résultats traduisent clairement l'influence de l'histoire des chargements successifs.

En chargement statique en compression, appliqué après les chargements en tension, on n'observe aucune différence significative tant pour $Q_{\ell}$ que pour $Q_{F}$ : rien ne permet de conclure à un effet certain du sens de la sollicitation.

En chargement cyclique en tension, la transition entre l'état stable et la rupture apparait relativement brutale : pour des niveaux de chargement inférieurs à la rupture, la répétition des cycles ne produit pas d'accroissement appréciable des déplacements en tête (Fig. 12); lorsque la charge de rupture cyclique est atteinte, celle-ci intervient fréquemment après quelques dizaines de cycles seulement.

Dans les argiles silteuses normalement consolidées, le comportement du pieu, caractérisé par le frottement latéral mobilisé et le déplacement en tête, n'est pas significativement différent :

- sous charge statique ou cyclique de même niveau (Fig. 15)

- en tension et en compression (ou en compressiontension alternées).

Ce résultat apparaît tout à fait fondamental pour le dimensionnement des pieux battus dans les formations argileuses normalement consolidées, mais ne doit pas dissimuler la difficulté du choix du frottement latéral, fonction des conditions initiales après le battage, de la reconsolidation plus ou moins lente du sol après battage ou une tempête, c'est-à-dire de toute l'histoire des chargements du pieu.

Les variations de pression interstitielle mesurées à l'interface sol-pieu durant le chargement statique ou cyclique restent toujours faibles, à la limite de la précision de mesure ( $\Delta u \simeq 5 \mathrm{kPa}$ ) quelle que soit la nature des sols concernés par les expérimentations (silts et sables lâches, argile silteuse très plastique). Ce résultat justifie la simulation numérique du comportement des pieux sous charges cycliques axiales en conditions drainées.

\section{3. - Comportement des pieux dans les formations carbonatées}

Les formations carbonatées, de granulométries et cimentations très variables, recouvrent de nombreuses zones d'activités pétrolières actuelles en mer (Golfe Persique, Australie, Côtes Ouest et Sud-Est de l'Inde, Méditerranée, Brésil, Caraïbes,...). le comportement mécanique des matériaux calcaires, fonction de leur origine détritique, chimique ou bio-construite, est caractérisé essentiellement par leur compressibilité et leur degré de cimentation. (Angemeer, 1973 et 1975). Les difficultés techniques rencontrées et les coûts de réalisation des fondations d'ouvrages en mers dans ces matériaux expliquent l'importance des travaux poursuivis depuis quelques années sur le comportement des pieux dans ces formations en vue d'aboutir à la définition de règles de l'art (Murff, 1985) applicables pour le choix et le dimensionnement des fondations.

\subsection{Pieux battus dans les sables carbonatés}

Le frottement latéral des pieux battus dans les sables carbonatés est plus faible que dans les sables siliceux : la différence est d'autant plus grande que la compressibilité du matériau est plus importante. La relation donnant le frottement latéral sol-pieu dans les sols frottants

$$
f=K p_{0}^{\prime} \operatorname{tg} \delta
$$

ne s'applique pas dans les sables carbonatés au-delà d'une valeur limite, atteinte, semble-t-il, à une profondeur plus faible que dans les sables siliceux.

Dans le cas du pieu battu sur le site de Plouasne
(Fig. 16), dans une formation carbonatée très compressible et peu cimentée (Fig. 5), le frottement latéral ne dépasse pas 1 à $2 \mathrm{kPa}$. (Nauroy et Le Tirant, 1985).

Des valeurs du frottement latéral de pieux dans diverses formations calcaires faiblement cimentées, rapportées dans la littérature, varient de 2 à $30 \mathrm{kPa}$. La dispersion importante des résultats s'explique au moins partiellement par les incertitudes sur les caractéristiques réelles des sables carbonatés difficilement identifiables sur des carottes toujours très remaniées. Les mesures in situ (par pénétrométrie et pressiométrie) et leur interprétation correcte apparaissent essentielles pour une meilleure prévision du comportement des pieux dans ces matériaux.

Les diverses hypothèses avancées (rupture des grains, degré de cimentation,...) ne fournissent pas d'explication satisfaisante aux faibles valeurs du frottement latéral observé. La recherche d'une relation encore la compressibilité des matériaux et le frottement latéral sol-pieu constitue probablement une voie prometteuse (Fig.17), mais de nombreuses informations bien identifiées devront être réunies avant d'aboutir à une validation acceptable pour les besoins de l'ingénierie.

Un dispositif d'ancrage à élément articulé (Luong et Habib, 1984), mis en place par battage ou vibrocarottage, applicable comme système d'ancrage pour des efforts modérés (inférieurs à une centaine de tonnes), présente, par rapport aux pieux battus, l'avantage de mobiliser des efforts importants de butée dans les formations carbonatées (sables coralliens par exemple), comme dans les autres matériaux. 


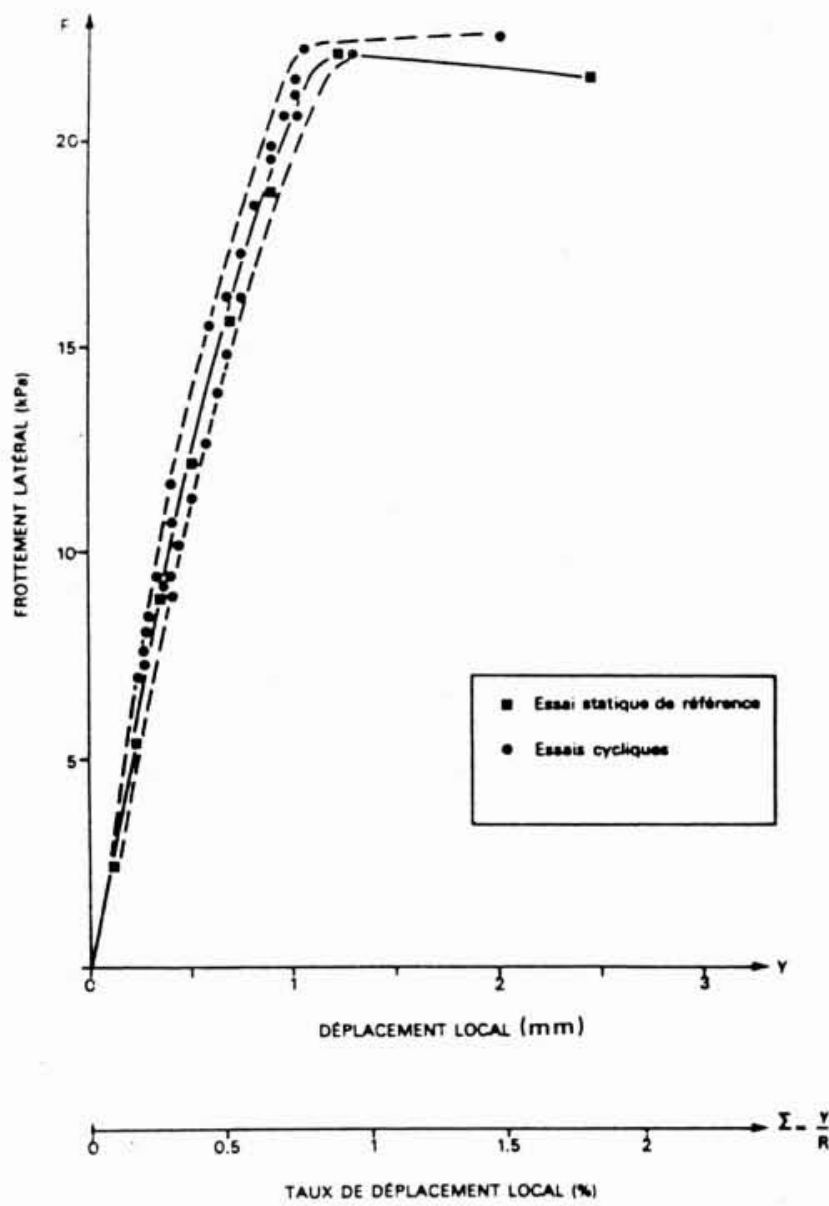

Figure 15. - Frottement latéral mobilisé sous charge statique et cyclique (argile silteuse de Cran).

Figure 17. - Frottement latéral sable-pieu en fonction de la compressibilité du sable.

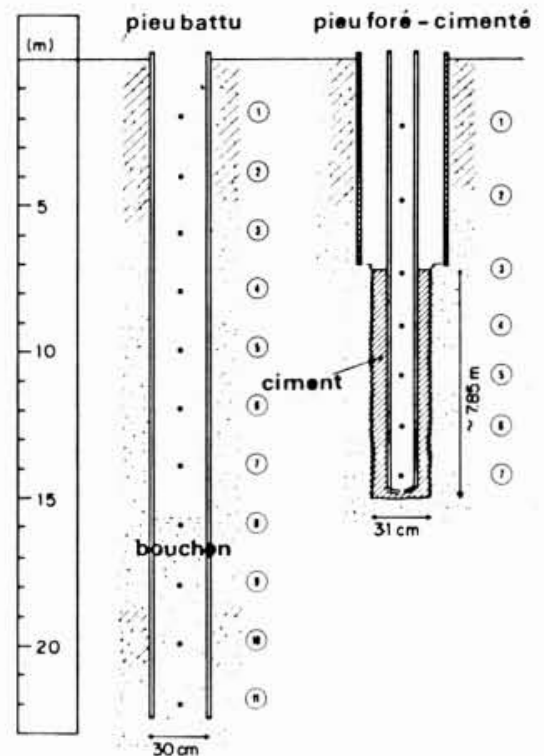

Figure 16. - Schémas des pieux battu et foré-cimenté mis en place à Plouasne.

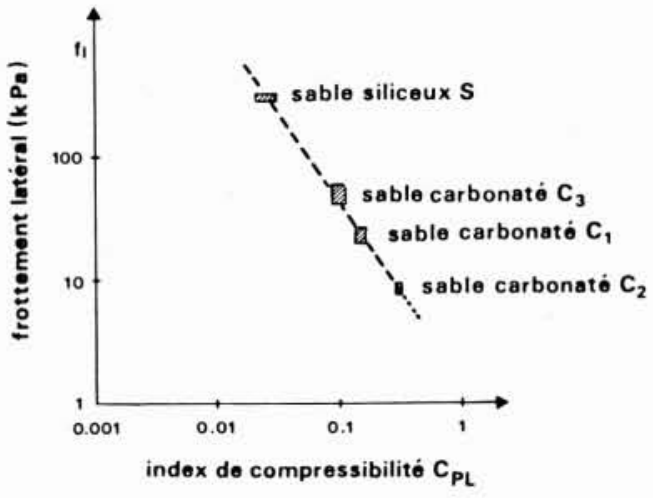

\subsection{Pieux forés-cimentés dans les sables carbonatés.}

Les très faibles valeurs du frottement latéral des pieux battus dans les sables carbonatés ont conduit à étudier le comportement des pieux forés-cimentés dans ces matériaux.

En chargement statique en tension, le frottement latéral mobilisé par des pieux forés-cimentés dans diverses formations carbonatées (Fig. 5 et 16) atteint et même dépasse $100 \mathrm{kPa}$ (Nauroy et Le Tirant, 1985). L'angularité des grains, leurs grandes dimensions et l'irrégularité des trous forés sont des facteurs qui accroissent la rugosité de la gaine de ciment entourant le tube et améliorent l'adhérence sol-ciment.

La compressibilité du matériau ne joue apparemment aucun rôle sur le frottement latéral mobilisé. Par contre, la cimentation contribue à accroître fortement le frottement latéral des pieux cimentés dans les niveaux très indurés ou rocheux (calcarénites ou calcaires durs par exemple).

En chargement cyclique en tension (Nauroy et al., 1985) :
- le comportement du pieu dépend à la fois de la charge moyenne $q_{\text {moy }}$ et de la charge maximale $q_{\max }$, c'est-à-dire de l'amplitude $\Delta q$ de la charge cyclique. Pour une même charge maximale, le déplacement du pieu peut tendre soit vers la stabilisation, soit vers la rupture suivant la valeur de $\Delta q$. Ce résultat bien établi pour les pieux battus s'appliquent donc aussi aux pieux forés-cimentés dans les formations carbonatées;

- la distribution de charge le long du pieu varie sous l'effet du cyclage. Pour les chargements cycliques n'affectant pas la stabilité du pieu, on observe, comme pour les pieux battus dans les formations silteuses ou argileuses, un transfert de charge de la partie supérieure vers la partie inférieure du pieu.

Les déboires rencontrés sur plusieurs sites de formations carbonatées pour la fixation de plates-formes treillis, où la capacité portante des pieux battus n'excède pas $25 \%$ de celle prévue, ont incité à proposer des solutions de " réparation" des pieux par injection de ciment. Des expérimentations sont actuellement poursuivies en ce sens sur les possibilités de réalisation et leur efficacité pour la mobilisation d'un frottement latéral. 


\section{Conclusion}

1) Dans le cas des pieux battus dans les formations silteuses et argileuses normalement consolidées (travaux de l'ARGEMA) ou surconsolidées (diverses recherches à l'étranger - Karksrud and Haugen, 1985), les connaissances actuelles constituant des bases solides pour l'amélioration du dimensionnement des pieux de fondation ou d'ancrage des ouvrages en mer. Les informations acquises notamment sur la dégradation cyclique permettent d'envisager dans un proche avenir une meilleure évaluation de la sécurité de ces ouvrages.

2) Dans le cas des formations carbonatées les résultats déjà obtenus sur le comportement des pieux battus et forés-cimentés représentent un acquis important, mais encore très insuffisant pour parvenir à des règles fiables de dimensionnement.

3) Des guides pratiques pour la conception et l'installation des pieux de fondations des ouvrages en mer sont en cours de préparation par l'ARGEMA. Parallèlement des travaux complémentaires sont poursuivis afin de suggérer ou promouvoir de nouvelles solutions aux problèmes de fondations difficiles, dans le cas des formations carbonatées notamment.

\section{Références}

Angemeer J., Carlson C.D. and Klick J.H. (1963). - Techniques and results of offshore pile load testing in calcareous soils. Proc. Sth Off. Tech. Conf., Houston, Paper OTC 1894.

Angemeer J., Carlson C.D., Stroud S. ans Kurzeme M. (1975). - Pile load tests in calcareous soils, conducted in 400 feet of water from a semi-submersible exploration. Proc. 7th Off. Tech. Conf., Houston, Paper OTC 2311.

Boulon M. et Foray P. (1985). - Simulation physique et numérique du comportement des fondations et ancrages en sols marins. Colloque d'Hydrotechnique, Grenoble, ( $\mathrm{La}$ Houille blanche, $\mathrm{n}^{\circ} 4 / 5-1986, \mathrm{p} . \quad$ ).

HOEG K. (1982). - Geotechnical issues in offshore engineering - State-of-the-Art Report. 3rd Int. Conf. on Behaviour of Off shore Structures, BOSS'82, MIT.

KARLSRUD K. and HAUGen T. (1985). - Behaviour of piles in clay under cyclic axial loading. Results of field model tests. $4 \mathrm{th} \mathrm{Int}$. Conf. on Behaviour of Offshore Structures, BOSS 85 . Delft, p. 589-600.

LE TiRant P. (1979), - Comportement des fondations et des ancrages de structures marines sous l'effet de sollicitations cycliques. Rev'ue de l'Institut Français du Pétrole, Vol. XXXIV, $\mathrm{n}^{\circ} 5$, p. 687-717.

LuONG M.P. et HABIB P. (1984). - Dispositif d'ancrage à élément articulé. Demande de brevet.
MURFF J.M. (1985). - Pile capacity in calcareous sands. State-ofthe-Art. Rapport présenté à la session spéciale Fondations offshore au $X I^{e}$ ICSMFE. San Francisco; to be submitted to the ASCE Journal of Geotechnical Eng.

Puech A. and Jezequel J.F. (1980). The effects of long time cyclic loadings on the behavior of a tension pile. Proc. 12th Off. Tech. Conf., Houston, Paper OTC 3870.

PUeCH A. (1982). - Basic data for the design of tension piles in silty soils. 3rd Int. Conf. on Behaviour of Offshore Structures. BOSS'82, MIT, Vol. 1, p. 141-157.

Puech A., Boulon M. and Meimon Y. (1982). - Tension piles: field data and numerical modelling. 2nd Int. Conf. on Numerical Methods in Offshore Piling. The Unir. of Texas, Austin.

Nauroy J.-F. and Le TiRant P. (1985). - Driven piles and drilled and grouted piles in calcareous sand. Proc. 17th Off. Tech. Conf., Houston. Paper OTC 4850.

Nauroy J.-F., Brucy F. and Le TIRANT P. (1985). - Static and cyclic load tests on a drilled and grouted pile in calcareous sand. 4th Int. Conf. on Behaviour of Offshore Structure. BOSS'85, Delft.

Nauroy J.-F., Brucy F. et Le Tirant P. (1985). - Pieux battus sollicités en tension. Comptes rendus du XI ICSMFE, San Francisco, Vol. 3, p. 1607-1610. 